This is a postprint version of:

Morillo, F. (2020). Is open access publication useful for all research fields? Presence of

funding, collaboration and impact. Scientometrics, 125(1), 689-716.

The final publication is available at Springer via https://doi.org/10.1007/s11192-020-03652-w

\title{
Is Open Access publication useful for all research fields? Presence of funding, collaboration and impact
}

\author{
Fernanda Morillo \\ IFS, Spanish National Research Council (CSIC), Albasanz, 26-28, 28037 Madrid, Spain. \\ Email address: fernanda.morillo@cchs.csic.es
}

\begin{abstract}
Open access $(O A)$ to every research result supported by funding bodies is a medium to long-term goal. This work aims to find out whether OA publication is useful for all research fields, regardless of their specific features. As a sample, articles from the WoS databases from two disparate disciplines (one from SSCl and another from SCIE) are selected, and several hypotheses related to the presence of funding acknowledgements, cooperation and citations are tested. A first look at the general distribution of publications shows that collaborative and funded research obtains a higher proportion of cited articles, and that this proportion increases in the case of OA publications. Moreover, the logistic regression reveals that the probability of finding an OA publication is significantly increased in the SCIE discipline, and by the presence of EU funding, international collaboration and citations. This probability rises with some interactions (e.g. presence of international funding and international collaboration, or international funding and citations). Regarding OA types, Green OA publications are the most related to fund recognition, although Gold OA/Bronze OA articles in international collaboration are also significantly related to financing. Concerning impact, the most likely cited OA type is the Hybrid OA. However, if papers include funding acknowledgements, the Bronze OA and Green OA publications increase their citation likelihood. Similarly, when Gold $\mathrm{OA}$ articles include international collaboration, there is a greater chance of citation. With these findings, it is possible to venture that OA publishing will be useful for all research fields, although their specific features should be considered. Consequently, funders should be aware of these particularities to stimulate OA without compromising the quality of the research.
\end{abstract}

\section{Keywords}

Open Access; Funding Acknowledgements; Collaboration; Impact; Research fields; WoS

\section{Introduction}

Since the beginning of the 21st century, different initiatives have emerged to favour open access $(O A)$ to scientific publications. In this context, funding bodies increasingly demand this type of access to publications derived from their sponsored research. The need for OA publication not only has to do with a general right to access knowledge, but is also believed to be related to the greater impact and progress of research. A better access to information will allow advancing in new knowledge based on the existing one (Gargouri et al. 2010; Larivière and Sugimoto 2018, Piwowar et al. 2018, Sotudeh and Estakhr 2018, Van Vlokhoven 2019). 
This is a postprint version of:

Morillo, F. (2020). Is open access publication useful for all research fields? Presence of

funding, collaboration and impact. Scientometrics, 125(1), 689-716.

The final publication is available at Springer via https://doi.org/10.1007/s11192-020-03652-w

Moreover, the growing cost of large toll-access publishers has made different institutions to resort to the OA to maintain their access to essential literature (Piwowar et al. 2018). In this way, the authors can retain control over the integrity of their work and the right to be duly recognised and cited (Martín-Martín et al. 2018). Among the initiatives of OA that have emerged more recently, it is worth mentioning the Plan $S^{1}$. This plan is based on a coalition of research funding organisations from different countries, with the support of the European Commission (EC) and the European Research Council (ERC). It is committed to implementing, from $2021^{2}$ onwards, the necessary measures to ensure that scientific results with funding from participating organisations are published in OA.

In this sense, the plans for the next Horizon Europe will include open science as the modus operandi. This will require not only scientific publications in $\mathrm{OA}$, but also research data in $\mathrm{OA}^{3}$. With regard to the definition of $O A$, although some define it as any free content for reading and reuse, in line with the CC-BY license, others are less strict and consider it sufficient that there is free access to online reading (Piwowar et al. 2018). Further to this, since the beginning of $O A$, various options have emerged, ranging from self-archive in public repositories (Green OA) to full access offered by publishers (Gold OA) (Sotudeh and Estakhr 2018). In addition, many institutions have created repositories, available to researchers to ensure compliance with OA policies (Pinfield 2010). The Gold OA option has been the consequence of the adaptation of the editors to the $O A$ requirements of funders and their journals revenue generally comes from article processing charge (APC). According to Björk and Solomon (2015), the subscription prices of journals have not always been linked to scientific quality, while the APCs are more in line with the quality and services offered. However, there are Gold OA journals (also called Diamond OA or Platinum OA) that do not have APC, because they cover all publication costs. Nevertheless, quality publications without APC are not available in all fields. Perhaps for this reason, APC costs are also experiencing hyperinflation, which is not disappearing with competition among publishers (Khoo 2019). Regarding the option of self-archiving, it has been offered by many research institutions as a possible solution to the problem of the growing costs of journals (Martín-Martín et al. 2018). On the other side, the OA can be found through alternative channels that offer a free version of the manuscript (Bronze), generally read only. However, the license of these articles is not clear and access to them may only be for a limited time. Thus, there is a risk of disappearance, which can also be found in OA Green copies filed on personal web pages that sometimes violate copyright rules (Björk et al. 2014).

Nonetheless, large commercial publishers have chosen an intermediate access: the Hybrid OA publishing. In this way, restricted access journals allow authors to publish their articles openly after the corresponding APC. At first, all authors had to face the costs, being forced to use part of their funds of research projects. Subsequently, agreements have been established between some funding agencies and publishers, which cover OA fees so that researchers can publish their results openly and thus increasing this type of publications. This system is not without criticism, since for publishers it does not imply any risk: they keep the subscription income and they charge for each article published in OA. That is paying twice for the same service. There are, however, some success stories in which a publication has completed the transition,

\footnotetext{
${ }^{1}$ Retrieved July 20, 2020, from https://www.coalition-s.org/about.

${ }^{2}$ This is an extension by one year of the initial date 2020 (retrieved July 20, 2020, from https://www.coalition-s.org/rationale-for-the-revisions).

${ }^{3}$ Retrieved July 20, 2020, from https://www.openaire.eu/horizon-europe.
} 
This is a postprint version of:

Morillo, F. (2020). Is open access publication useful for all research fields? Presence of

funding, collaboration and impact. Scientometrics, 125(1), 689-716.

The final publication is available at Springer via https://doi.org/10.1007/s11192-020-03652-w

moving from Hybrid to Gold OA (e.g. Nature Communications) (Björk 2017, Björk and Solomon 2015, Martín-Martín et al. 2018, Pinfield 2010, Sotudeh and Estakhr 2018, Wang et al. 2015b). However, Plan $S^{\prime}$ principles entail that publication must be done in journals/platforms in compliant $O A$, which means that authors must retain their copyright and that Hybrid OA is not considered to fulfil this plan, although the plan also considers that funders can reach transitional agreements with Hybrid publishers, defining deadlines, always in order to achieve the full OA model.

In this context, the hazard of market concentration is even greater. Therefore, Khoo (2019) considers that it is necessary to re-examine the evolution of APC after the implementation of Plan S, to analyse whether the prices of academic publications have increased further. The plan states that when there are fees for publishing, the funders will take over, as long as fees are justifiable and proportional. However, Plan $\mathrm{S}$ does not establish what it considers justifiable and proportional (Else 2018). In fact, Khoo (2019) considers that there is no reasonable APC. Journals that achieve a certain prestige tend to increase costs based on the number of manuscripts they receive, since only accepted manuscripts have APCs. In addition, the APC implies that scientists are no longer free to decide where to publish because this depends on the funds they receive to meet these new costs (Perianes-Rodríguez and Olmeda-Gómez 2019). Moreover, the access to this system would be restricted to researchers with funds to pay for that space and, at the same time, many researchers would be forced to abandon well-established journals without any viable alternatives. On the other hand, an unwanted consequence of the APC-based OA could be that large publishers stop trying to attract excellent works, which are what give them prestige in the current subscription-based system. However, some studies point to the fact that if a journal loses prestige, authors may decide to send their manuscripts to other journals with more demanding acceptance criteria. This is because authors place greater importance on the quality, validity, impact and review process of a journal than on everything else (Khoo 2019, Sotudeh and Estakhr 2018, Van Vlokhoven 2019).

Given this scenario, various tools have been developed to analyse the growing corpus and have as much information as possible to assess the potential influence of the funders' recommendations in various research areas. On the one hand, several works have studied the results of the requirements of different funding agencies with respect to the open publication of their sponsored findings. In this respect, the paper by Larivière and Sugimoto (2018) presents an analysis of compliance with large-scale OA mandates, identifying more than 1.3 million documents, of which two-thirds are available for reading, although with significant variations between agencies. They conclude that when there are incentives and support, the researchers comply. On the other hand, studies such as Van Leeuwen et al. (2018) focus not only on identifying OA publications (mainly Gold OA), but also on analysing those publications in terms of impact. Their results present lower citation rates for publication in Gold OA, which they think it could be due to the recent incorporation of these journals to the scientific domain, combined perhaps with a less selective criterion of inclusion of new journals in the WoS databases. Nevertheless, in the study by Piwowar et al. (2018) they observed that, in general, OA usually receive more citations, having experimenting a significant growth in size and impact in the last decade. In an earlier study, Antelman (2004) already observes a greater impact for OA in four different disciplines. Similarly, Sotudeh and Estakhr (2018) find a sustained citation advantage over time among OA versus non-OA publications in all areas, although not all of them are equally benefited. 
This is a postprint version of:

Morillo, F. (2020). Is open access publication useful for all research fields? Presence of

funding, collaboration and impact. Scientometrics, 125(1), 689-716.

The final publication is available at Springer via https://doi.org/10.1007/s11192-020-03652-w

Apart from that, the citation advantage is usually used as recognition of the validity of OA publication and has been linked to the authors' selection of their best works for OA. However, the study by Sotudeh (2019) concludes that, if the authors' self-selectivity exists, it is not responsible for the OA citation advantage, at least with regard to the selection of topics. Besides, during her data collection, Antelman (2004) observed that it was usual for authors to publish all or none of their articles in OA. In summary, the OA allows users to decide what information they need and cite based on higher quality and not based on access, as also stated by Cintra et al. (2018). Further to citation advantage and differences by discipline, the existence of funding seems to be greater in the research published in OA. Indeed, as well as including open publishing requirements, some agencies offer support for APC (Wang et al. 2015a). On the other hand, some authors have found a relationship between cooperation and OA. This is the case of Valderrama-Zurián et al. (2019), which analyse the OA publication in several disciplines of education (SSCl, period 2010-2016). In their set, they observe a greater international collaboration, especially in the Gold OA publication and assume that this cooperation makes it easier to find a partner that deals with APC. In this line, Breugelmans et al. (2018) study the relationship between impact and OA when there is international collaboration, and conclude that there is a citation advantage. The only exception they find is in the collaboration between sub-Saharan African countries.

The current study goal is to analyse the presence of similar outcome in disparate disciplines. In this way, it will be possible to evaluate the measures taken by the funding bodies and suggest additional measures to promote the share of information while maintaining the quality of the research carried out in different fields.

\section{Objectives}

This work aims to find out whether OA publication is useful for all research fields, regardless of their specific features. As a sample, articles from the WoS databases from two disparate disciplines (one from $\mathrm{SSCl}$ and another from SCIE) are selected, and several hypotheses are tested:

- It is presumed that, in general, there will be a higher proportion of funding acknowledgments (FA) in OA articles, mainly from international and EU sources.

- OA documents are supposed to present greater collaboration, especially of international scope.

- OA items are expected to obtain a citation advantage over non-OA articles, being the Gold OA type the least likely to be cited.

\section{Materials and Methodology}

Articles written in English in 2017 are selected, because WoS only collects FA when in English. A list of categories with the highest production in $\mathrm{SSCl}$ is obtained, choosing the second, Economics (Econ), and retrieving 20,030 items. The first one (Public, Environmental \& Occupational Health) is not chosen because is included in both SSCI and SCIE. Likewise, a list of categories is obtained in $\mathrm{SCIE}$, selecting one of similar size and with a greater proportion of $\mathrm{OA}$, Immunology (Immu), and retrieving 23,988 items. Besides, different characteristics of these WoS articles are analysed including number of authors, addresses, countries, references, pages 
This is a postprint version of:

Morillo, F. (2020). Is open access publication useful for all research fields? Presence of

funding, collaboration and impact. Scientometrics, 125(1), 689-716.

The final publication is available at Springer via https://doi.org/10.1007/s11192-020-03652-w

and citations, usage count, $\mathrm{OA}$ and FA. Addresses and countries are used to reveal the existence of collaboration and to separate documents with only national collaboration (Nat Coll) from those that include international collaboration (Int Coll). Furthermore, to allow comparisons between research fields, a citation ranking is carried out, ranging from the absence of citation to highly cited articles. These citations were obtained in mid-2019, so there is a 1.5 to 2.5 -year citation window. However, this allows assessing the presence of early citations in $\mathrm{OA}$ articles, which is a characteristic feature of this type of publication. On the other hand, the usage count offers complementary information as a measure of potential interest of the published research. This value represents the number of times a WoS record has been saved or the number of times the full text has been accessed since February 1, 2013 . Some authors (e.g. Chi et al. 2019) have observed that there is certain correlation with other measures of research impact, so it is interesting to analyse the influence of this indicator on OA publications.

Taking into account the OA characteristic of WoS articles, the different types are also studied to be able to highlight differences, avoiding duplications. Therefore, in case of concurrence, Gold access prevails, followed by Hybrid, Bronze and Green OA. These are based on WoS multiple versions of $\mathrm{OA}^{5}$ : DOAJ Gold, Other Gold (Hybrid), Bronze and Green OA. For articles in Green $O A$, they link to peer-reviewed versions hosted legally in open repositories, both the accepted version and the published version. For all articles in OA, WoS give preference to Gold or Bronze versions when available. On the other hand, these databases make weekly updates to include new OA links and any changes in their status, taking into account everything that Impactstory finds. This is possible because the owner of WoS, Clarivate Analytics, collaborates with Impactstory, a non-profit organisation whose goal is to make the OA content easier to find. For this reason, these databases use Impactstory's improved OA identification technology.

With respect to FA, the WoS articles that include acknowledgements to any financial support are identified. Additionally, the different types of funding sources are analysed, separating the documents that acknowledge European funds $\left(E U F A^{6}\right)$ from those that include funds from two or more countries (Int FA) and from those that only include national funds (Nat FA). For the identification of the FA types, this study has used the automation processes previously developed by Morillo and Álvarez-Bornstein (2018). These processes analyse documents with data in the FA field and extract the main funding bodies along with their place of origin, whenever possible. In the present paper, only the geographical location is processed to distinguish between articles supported by national, international or EU sources.

Further to this, statistical tests are used to reveal which characteristics are associated with an $\mathrm{OA}$ article. In particular, the aim is to verify whether the publications in OA present greater FA, collaboration and citation, as well as to determine the differences between fields and between OA types.

\footnotetext{
${ }^{4}$ Retrieved July 20, 2020, from https://images.webofknowledge.com/images/help/WOS/hp usage score.html.

${ }^{5}$ Retrieved July 20, 2020, from https://images.webofknowledge.com/images/help/WOS/hp whatsnew wos.html.

${ }^{6}$ European Structural \& Investment Funds (ESIF) are not considered for the EU FA type because the EU countries administer the funds on a decentralised basis through shared management (retrieved July 20, 2020, from https://ec.europa.eu/eip/ageing/funding/ESIF en).
} 
This is a postprint version of:

Morillo, F. (2020). Is open access publication useful for all research fields? Presence of

funding, collaboration and impact. Scientometrics, 125(1), 689-716.

The final publication is available at Springer via https://doi.org/10.1007/s11192-020-03652-w

\section{Logistic regression}

Several logistic regression models are applied with all the variables of interest, obtaining both the main effects and the interactions between them. In order to determine the goodness of fit of the models, the Nagelkerke pseudo-R2 statistic is used. Finally, the Wald test is applied to calculate the statistical significance of the model parameters, establishing a p-value less than 0.05. To avoid multicollinearity problems, the number of authors, addresses, countries and pages are excluded. Descriptive statistics of dependent and independent variables are shown in Table 1 and Table 2. Logistic regression models are constructed with the presence/absence of $\mathrm{OA}$ as the dependent variable (Table 3 ). That is, the probability of finding a WoS article published in OA, based on selected independent variables, which include FA types, collaboration types, citation quartiles, number of references, usage count and field (Immu vs Econ). Likewise, similar models are applied using the presence/absence of FA as the dependent variable (Table 4), with OA types, collaboration types, citation quartiles, number of references, usage count and field (Immu vs Econ) as independent variables. Finally, logistic regression models are constructed with the presence/absence of citations as the dependent variable (Table 5), including OA types, FA types, collaboration types, number of references, usage count and field (Immu vs Econ) as independent variables. Furthermore, the interactions between several variables are also evaluated and their influence on the different dependent variables (see Appendix).

\section{Results}

\section{General Data}

A preliminary analysis of the data reveals the differences between the selected disciplines, not only in terms of the presence of publications in OA ( $50 \%$ in Immu and less than $15 \%$ in Econ), but also in terms of FA ( $79 \%$ vs. $48 \%)$. These figures are related to the characteristic traits of each research field, as well as those observed in collaboration (85\% in Immu versus $67 \%$ in Econ) and in citations (85\% versus 64\%). In the case of collaboration, it should be taken into account that, after processing the information of the two selected disciplines, it is observed that there are 100 articles whose addresses or authorship are unknown. For this reason, logistic regression models only analyse 43,918 items (Table 1).

On the other hand, the detailed information offered in Table 1 for OA types allows to see that publications in Econ are disseminated mainly in Bronze OA, while for Immu Gold OA is the most frequent. Besides, international collaboration is $52 \%$ of the total collaboration for Econ and less than $36 \%$ for Immu. As for citations, they have been distributed as evenly as possible to allow comparisons. Therefore, for Econ, the highly cited category includes articles cited at least 3 times, while for Immu, this category comprises articles cited 7 times or more. Finally, with respect to the continuous variables, Immu stands out for its higher number of references, while Econ has a greater usage count (Table 2). 
This is a postprint version of:

Morillo, F. (2020). Is open access publication useful for all research fields? Presence of funding, collaboration and impact. Scientometrics, 125(1), 689-716.

The final publication is available at Springer via https://doi.org/10.1007/s11192-020-03652-w

Table 1. Dependent and independent variables used in logistic regression models

\begin{tabular}{|c|c|c|c|c|c|c|c|c|}
\hline \multirow[b]{2}{*}{ Access } & \multirow[b]{2}{*}{ No OA } & & \multicolumn{2}{|c|}{ Econ } & \multicolumn{2}{|c|}{ Immu } & \multicolumn{2}{|c|}{ Total Art } \\
\hline & & & 17129 & $85.5 \%$ & 11910 & $49.6 \%$ & 29039 & $66.0 \%$ \\
\hline & $\mathrm{OA}$ & Green OA & 601 & $3.0 \%$ & 1671 & $7.0 \%$ & 2272 & $5.2 \%$ \\
\hline & & Bronze OA & 910 & $4.5 \%$ & 3775 & $15.7 \%$ & 4685 & $10.6 \%$ \\
\hline & & Hybrid OA & 879 & $4.4 \%$ & 1458 & $6.1 \%$ & 2337 & $5.3 \%$ \\
\hline & & Gold OA & 511 & $2.6 \%$ & 5174 & $21.6 \%$ & 5685 & $12.9 \%$ \\
\hline & & Total OA & 2901 & $14.5 \%$ & 12078 & $50.4 \%$ & 14979 & $34.0 \%$ \\
\hline & Total Art & & 20030 & $100.0 \%$ & 23988 & $100.0 \%$ & 44018 & $100.0 \%$ \\
\hline \multirow[t]{6}{*}{ Funding } & No FA & & 10445 & $52.1 \%$ & 5073 & $21.1 \%$ & 15518 & $35.3 \%$ \\
\hline & FA & Nat FA & 7594 & $37.9 \%$ & 14897 & $62.1 \%$ & 22491 & $51.1 \%$ \\
\hline & & Int FA & 1246 & $6.2 \%$ & 2931 & $12.2 \%$ & 4177 & $9.5 \%$ \\
\hline & & EU FA & 745 & $3.7 \%$ & 1087 & $4.5 \%$ & 1832 & $4.2 \%$ \\
\hline & & Total FA & 9585 & $47.9 \%$ & 18915 & $78.9 \%$ & 28500 & $64.7 \%$ \\
\hline & Total Art & & 20030 & $100.0 \%$ & 23988 & $100.0 \%$ & 44018 & $100.0 \%$ \\
\hline \multirow[t]{6}{*}{ Collaboration } & No Coll & & 6608 & $33.1 \%$ & 3572 & $14.9 \%$ & 10180 & $23.2 \%$ \\
\hline & Coll & Nat Coll & 6401 & $32.1 \%$ & 13115 & $54.7 \%$ & 19516 & $44.4 \%$ \\
\hline & & Int Coll & 6944 & $34.8 \%$ & 7278 & $30.4 \%$ & 14222 & $32.4 \%$ \\
\hline & & Total Coll & 13345 & $66.9 \%$ & 20393 & $85.1 \%$ & 33738 & $76.8 \%$ \\
\hline & Total Art & vith data & 19953 & $100.0 \%$ & 23965 & $100.0 \%$ & 43918 & $100.0 \%$ \\
\hline & (No data) & & 77 & & 23 & & 100 & $0.2 \%$ \\
\hline \multirow{6}{*}{$\begin{array}{l}\text { Citation } \\
\text { quartiles* }\end{array}$} & No cited & & 7306 & $36.5 \%$ & 3498 & $14.6 \%$ & 10804 & $24.5 \%$ \\
\hline & Cited & Low cited & 4579 & $22.9 \%$ & 7181 & $29.9 \%$ & 11760 & $26.7 \%$ \\
\hline & & Medium cited & 2731 & $13.6 \%$ & 7547 & $31.5 \%$ & 10278 & $23.3 \%$ \\
\hline & & Highly cited & 5414 & $27.0 \%$ & 5762 & $24.0 \%$ & 11176 & $25.4 \%$ \\
\hline & & Total Cited & 12724 & $63.5 \%$ & 20490 & $85.4 \%$ & 33214 & $75.5 \%$ \\
\hline & Total Art & & 20030 & $100.0 \%$ & 23988 & $100.0 \%$ & 44018 & $100.0 \%$ \\
\hline
\end{tabular}

* Citations have been distributed as evenly as possible to allow comparisons. Low cited: 1 citation for Econ, 1-2 citations for Immu. Medium cited: 2 citations for Econ, 3-6 citations for Immu. Highly cited: >=3 citations for Econ, >=7 citations for Immu.

Table 2. Continuous independent variables used in logistic regression models

\begin{tabular}{llrrr}
\hline & & Econ & \multicolumn{1}{c}{ Immu } & \multicolumn{1}{c}{ Total } \\
\hline References & Mean & 44.15 & 51.67 & 48.25 \\
& Median & 40.00 & 42.00 & 41.00 \\
\multirow{3}{*}{ Usage count } & Percentile 75 & 56.00 & 59.00 & 57.00 \\
& Mean & 10.26 & 6.21 & 8.05 \\
& Median & 7.00 & 4.00 & 5.00 \\
& Percentile 75 & 13.00 & 8.00 & 10.00 \\
\hline
\end{tabular}

If the general distribution of publications is analysed, it is possible to observe that collaborative and funded research obtains a higher proportion of cited articles (Figure 1). In the case of OA items, it seems that this proportion increases, so it is particularly interesting to study which are the most significant variables for this type of publication. 
This is a postprint version of:

Morillo, F. (2020). Is open access publication useful for all research fields? Presence of funding, collaboration and impact. Scientometrics, 125(1), 689-716.

The final publication is available at Springer via https://doi.org/10.1007/s11192-020-03652-w

Figure 1. Percentage of cited articles for OA by FA and collaboration

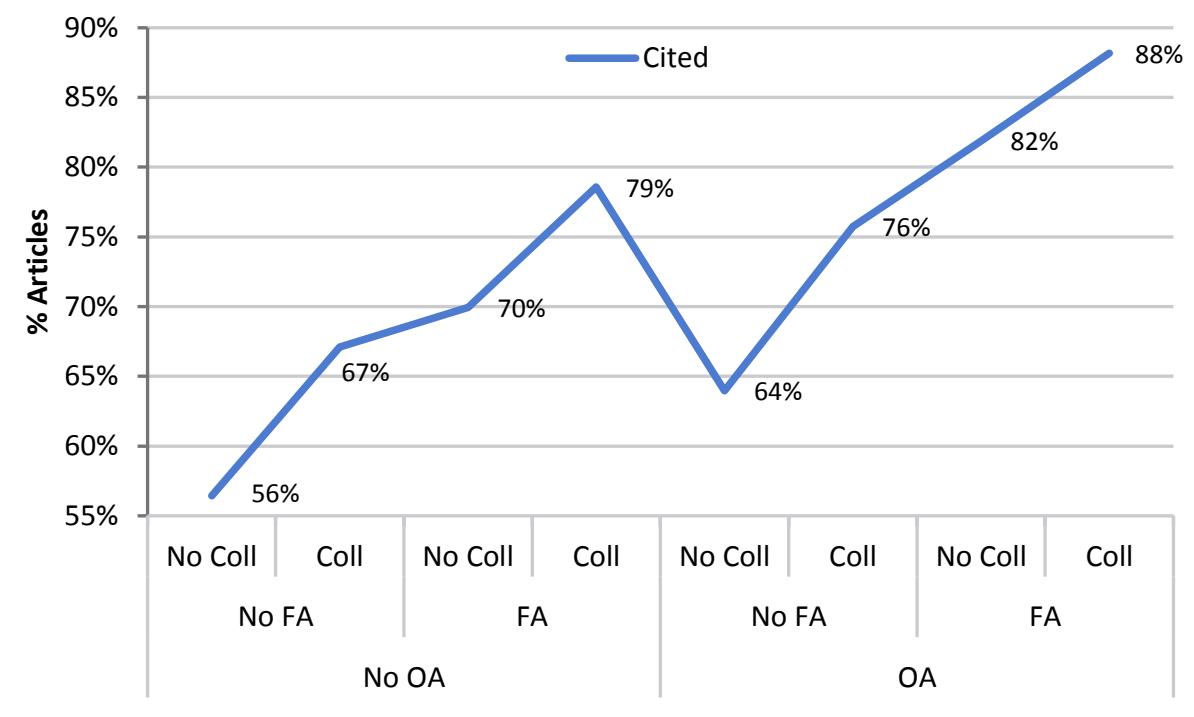

\section{Logistic regression}

With the aim to find out what characteristics OA publications have, several logistic regression models are built. Using the OA presence/absence as the dependent variable, which represents $34 \%$ vs $66 \%$ of the articles (Table 1), its best explanatory variables are the existence of FA (mainly from the EU sources), international collaboration, highly cited publications and Immu research. This is shown in Table 3, which presents a Nagelkerke's R2 of 0.243 , an OR 2.88 $(p<0.001)$ for OA publications with EU FA, great international collaboration (OR 1.35, $\mathrm{p}<0.001$ ), highly cited research (OR $1.84, \mathrm{p}<0.001$ ) and a larger representation of the Immu field (OR of 4.54, $p<0.001$ ).

Table 3. Logistic regression for the OA presence/absence

\begin{tabular}{lcccc}
\hline & \multicolumn{4}{c}{ LR for OA } \\
\cline { 2 - 5 } & B & S.E. & Sig. & OR \\
\hline No FA vs & & & & \\
$\quad$ Nat FA & 0.651 & 0.027 & 0.000 & 1.918 \\
$\quad$ Int FA & 0.817 & 0.042 & 0.000 & 2.264 \\
$\quad$ EU FA & 1.058 & 0.057 & 0.000 & 2.880 \\
No Coll vs & & & & \\
$\quad$ Nat Coll & 0.010 & 0.031 & 0.742 & 1.010 \\
$\quad$ Int Coll & 0.297 & 0.033 & 0.000 & 1.346 \\
No cited vs. & & & & \\
$\quad$ Low cited & 0.056 & 0.034 & 0.098 & 1.057 \\
$\quad$ Medium cited & 0.317 & 0.035 & 0.000 & 1.374 \\
$\quad$ Highly cited & 0.610 & 0.036 & 0.000 & 1.841 \\
References & 0.002 & 0.000 & 0.000 & 1.003 \\
Usage count & -0.026 & 0.002 & 0.000 & 0.974 \\
Immu vs Econ & 1.513 & 0.027 & 0.000 & 4.539 \\
Constant & -2.382 & 0.039 & 0.000 & 0.092 \\
Nagelkerke R Square & 0.243 & & & \\
\hline$\quad$ OR odds ratio = Exp(B) & & & &
\end{tabular}


This is a postprint version of:

Morillo, F. (2020). Is open access publication useful for all research fields? Presence of funding, collaboration and impact. Scientometrics, 125(1), 689-716.

The final publication is available at Springer via https://doi.org/10.1007/s11192-020-03652-w

Furthermore, the likelihood of finding an OA publication rises with the presence of some interactions (see Appendix). For instance, Figure 2 shows how the mean predicted probability for $\mathrm{OA}$ increases with the synergy between funding and collaboration. This connection is particularly strong and significant with international funding and international collaboration (OR 1.71, $\mathrm{p}<0.001$ ). In addition, there are also interactions between the presence of funding and citations (Figure 3), being significant those that occur with international (OR 1.49, p <0.001) and only national sources, but not those that occur with EU FA, with high presence in OA publications, regardless of citations. Moreover, it is interesting to note the importance of the synergy between funding and Immu, especially with the international FA (OR 1.93, p <0.001), possibly explained by the large proportion of documents with this type of funds in this discipline (Figure 4). Likewise, the probability of finding an OA publication increases with the international collaboration in Immu (see Appendix).

Figure 2. Mean predicted probability for OA by FA Types and Coll Types

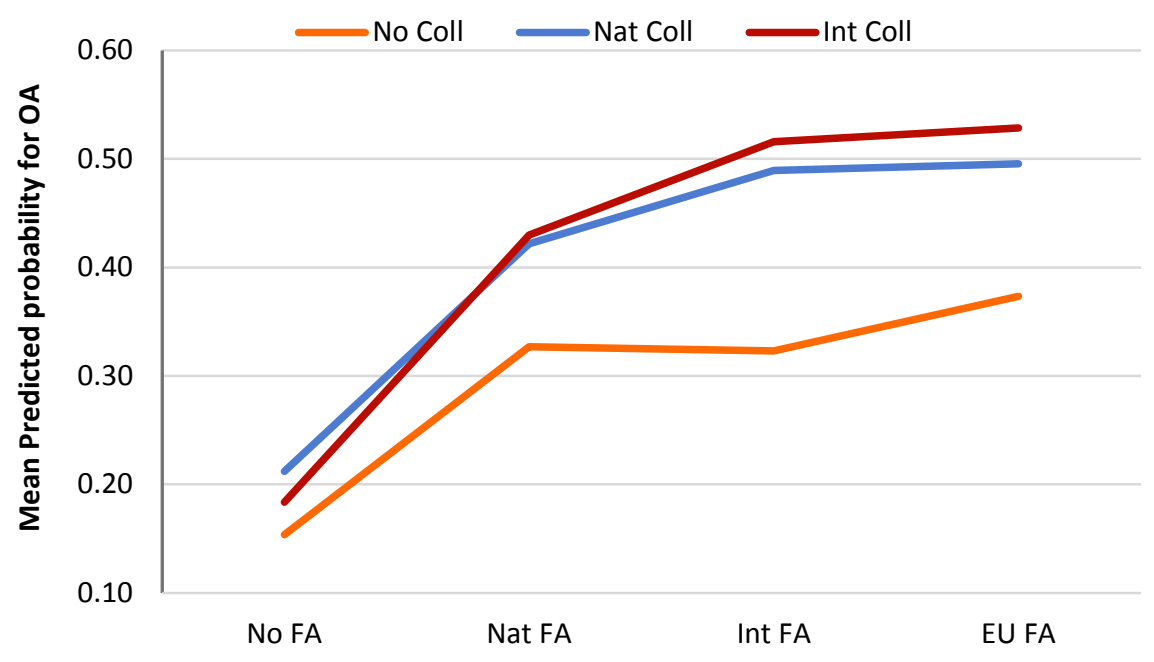

Figure 3. Mean predicted probability for OA by FA Types and No cited/Cited

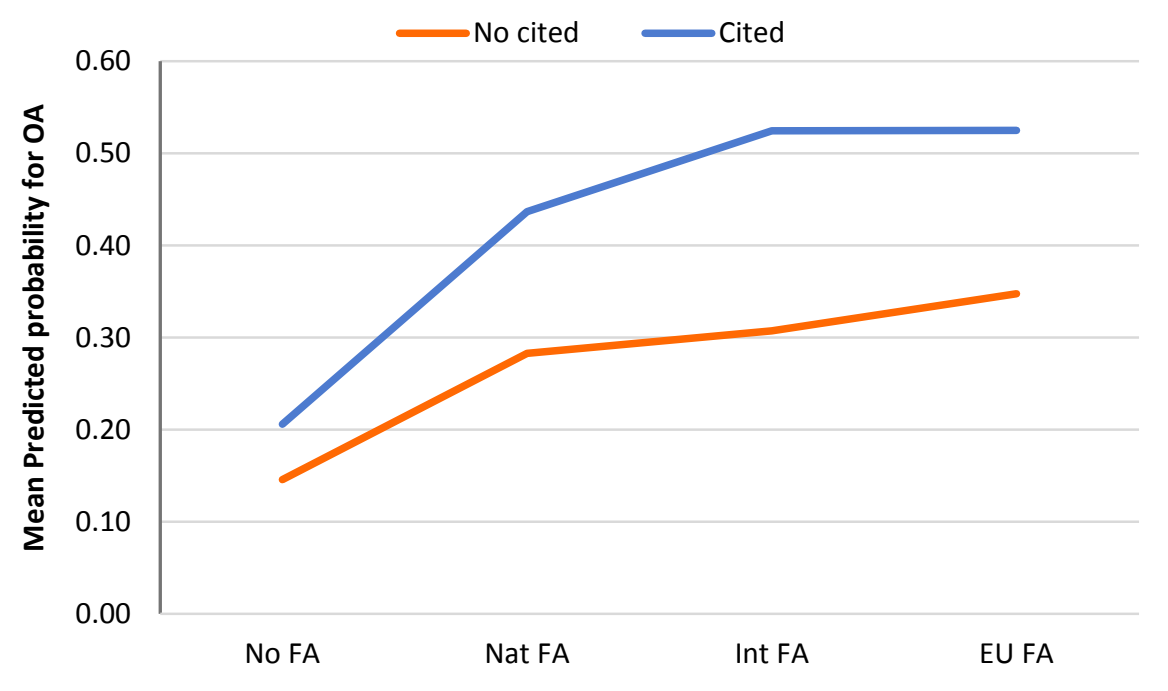


This is a postprint version of:

Morillo, F. (2020). Is open access publication useful for all research fields? Presence of funding, collaboration and impact. Scientometrics, 125(1), 689-716.

The final publication is available at Springer via https://doi.org/10.1007/s11192-020-03652-w

Figure 4. Mean predicted probability for OA by FA Types and Field

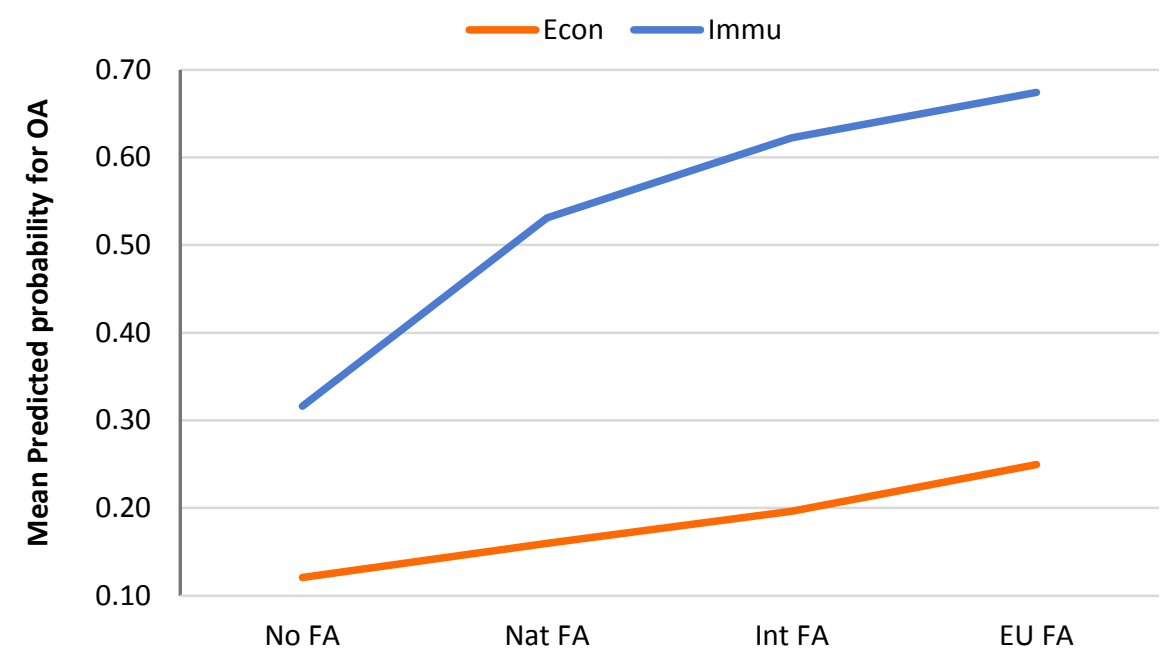

Considering that funding seems decisive to OA publishing, an additional analysis is performed with the dependent variable of the FA presence/absence, which represents $65 \%$ vs $35 \%$ of the articles (Table 1). Among other things, its relation with the different OA types is studied. The results show that the overall probability of finding FA in publications (Table 4) increases especially in Green OA items (OR 2.65, $p<0.001$ ), in those with international collaboration (OR $2.25, \mathrm{p}<0.001$ ), with high citations (OR 1.60, $\mathrm{p}<0.001$ ) and in Immu field (OR 3.19, $\mathrm{p}<0.001$ ).

Table 4. Logistic regression for the FA presence/absence

\begin{tabular}{lcccc}
\hline & \multicolumn{4}{c}{ LR for FA } \\
\cline { 2 - 5 } & B & S.E. & Sig. & OR \\
\hline No OA vs & & & & \\
$\quad$ Green OA & 0.976 & 0.062 & 0.000 & 2.654 \\
$\quad$ Bronze OA & 0.769 & 0.042 & 0.000 & 2.157 \\
$\quad$ Hybrid OA & 0.776 & 0.056 & 0.000 & 2.172 \\
$\quad$ Gold OA & 0.489 & 0.038 & 0.000 & 1.631 \\
$\quad$ No Coll vs & & & & \\
$\quad$ Nat Coll & 0.450 & 0.027 & 0.000 & 1.568 \\
$\quad$ Int Coll & 0.812 & 0.029 & 0.000 & 2.252 \\
$\quad$ No cited vs & & & & \\
$\quad$ Low cited & 0.223 & 0.030 & 0.000 & 1.250 \\
$\quad$ Medium cited & 0.428 & 0.033 & 0.000 & 1.534 \\
$\quad$ Highly cited & 0.471 & 0.033 & 0.000 & 1.601 \\
References & & & & \\
Usage count & 0.013 & 0.001 & 0.000 & 1.013 \\
Immu vs Econ & 1.160 & 0.025 & 0.000 & 3.191 \\
$\quad$ Constant & -0.987 & 0.028 & 0.000 & 0.373 \\
$\quad$ Nagelkerke R Square & 0.206 & & & \\
\hline$\quad$ OR odds ratio = Exp(B) & & & &
\end{tabular}

Besides, the likelihood of finding FA in publications rises with the interaction between different variables (see Appendix). On the one hand, Figure 5 presents how some connections between $\mathrm{OA}$ types and collaboration types increases the mean predicted probability for FA, being 
This is a postprint version of:

Morillo, F. (2020). Is open access publication useful for all research fields? Presence of funding, collaboration and impact. Scientometrics, 125(1), 689-716.

The final publication is available at Springer via https://doi.org/10.1007/s11192-020-03652-w

significant those between Green OA publications and only national collaboration (OR 1.73, $p<0.001$ ), between Gold OA and international collaboration (OR 1.48, $p<0.001$ ), and between Bronze OA and international collaboration (OR 1.42, $p<0.005$ ). On the other hand, there are also interactions between OA types and citations, particularly with the Green OA publications (OR 1.55, $\mathrm{p}<0.005$ ) and with the exception of the Hybrid OA type, which is not significant (Figure 6). Finally, synergies can also be observed in Figure 7, between the OA types and Immu, being especially intense with the Green OA publications (OR 2.62, $p<0.001$ ).

Figure 5. Mean predicted probability for FA by OA Types and Coll Types

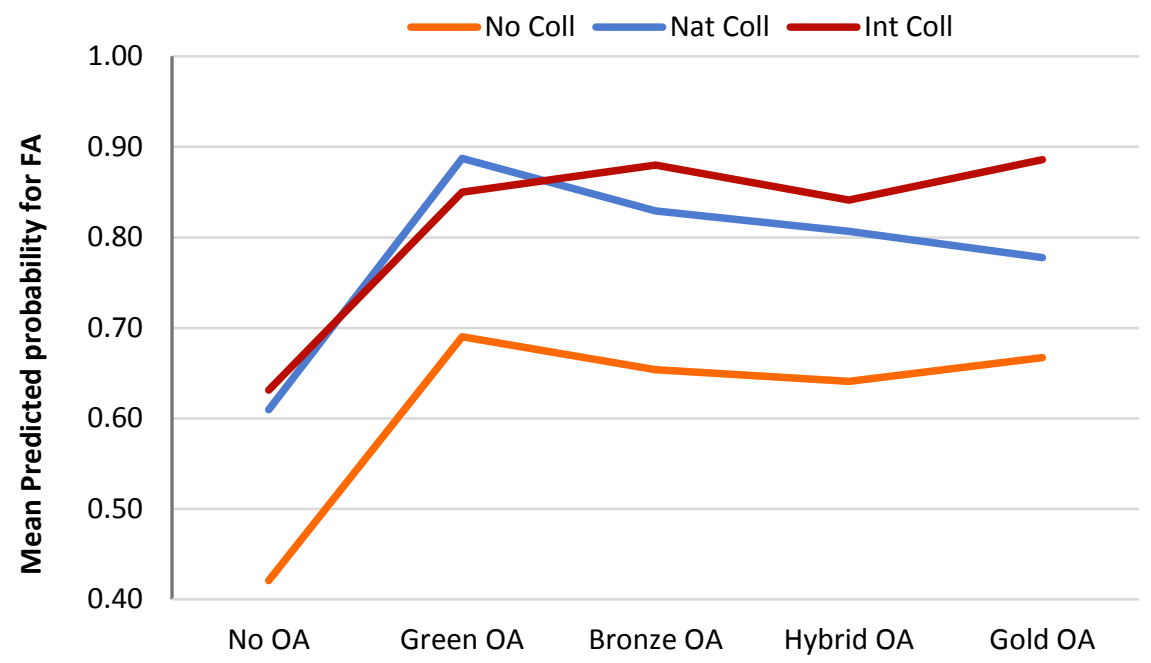

Figure 6. Mean predicted probability for FA by OA Types and No cited/Cited

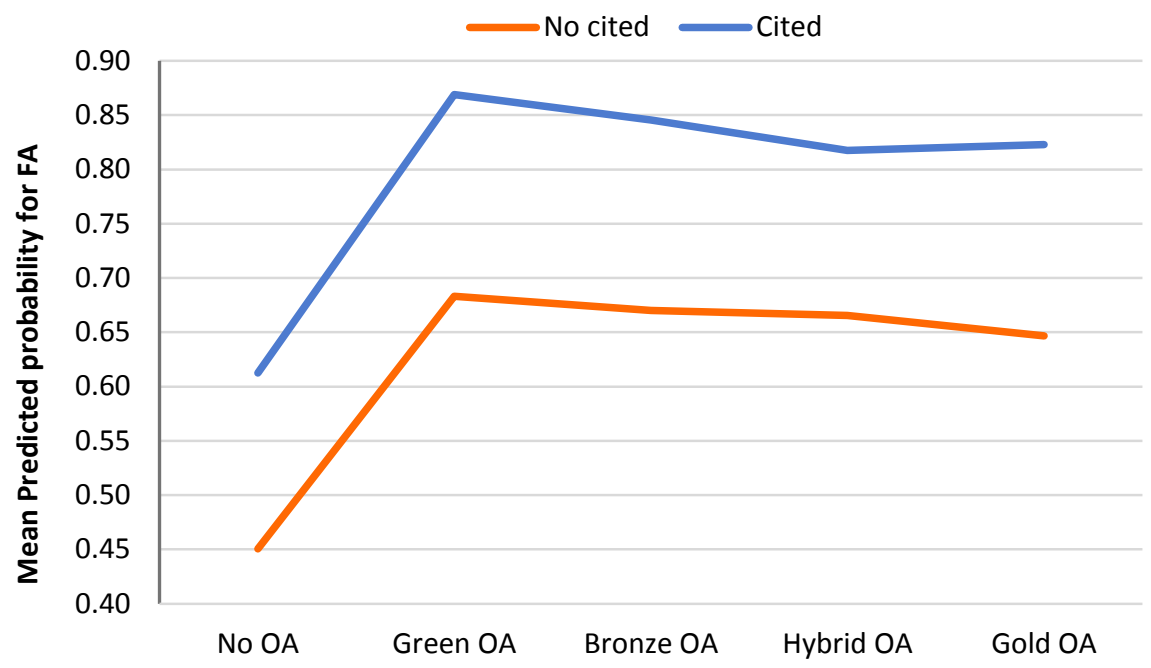


This is a postprint version of:

Morillo, F. (2020). Is open access publication useful for all research fields? Presence of funding, collaboration and impact. Scientometrics, 125(1), 689-716.

The final publication is available at Springer via https://doi.org/10.1007/s11192-020-03652-w

Figure 7. Mean predicted probability for FA by OA Types and Field

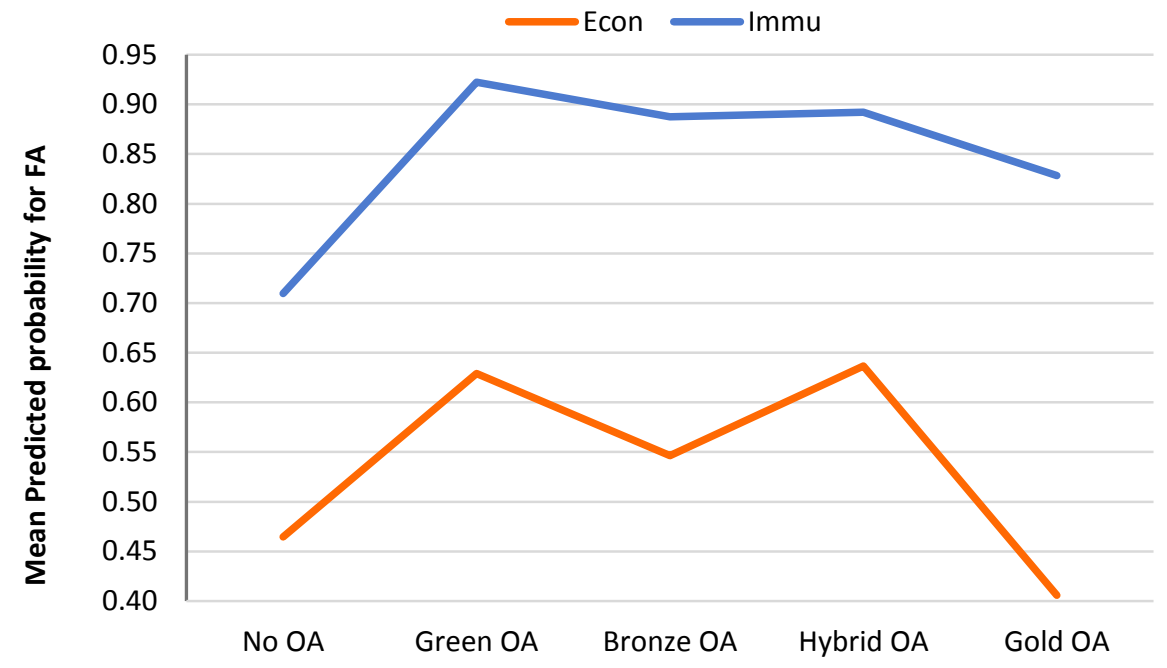

On the other hand, the citation variable is also examined to see its relationship with the different OA types. Articles that have been cited represent $75 \%$ of all the analysed articles (Table 1). If the presence/absence of citations is considered as the dependent variable (Table 5), a greater probability is observed for OA publications, particularly for Hybrid OA items (OR 1.79, $p<0.001$ ), with the exception of Gold OA, although this value is not significant. The likelihood of being cited increases also for funded publications, especially for EU FA (OR 1.89, $p<0.001$ ), for those that include collaboration (especially international, OR 1.46, $p<0.001$ ) and for those in Immu field (OR 3.60, p <0.001).

Table 5. Logistic regression for the presence/absence of citations

\begin{tabular}{lcccc}
\hline & \multicolumn{4}{c}{ LR for Cited } \\
\cline { 2 - 5 } & B & S.E. & Sig. & OR \\
\hline No OA vs & & & & \\
$\quad$ Green OA & 0.488 & 0.067 & 0.000 & 1.629 \\
$\quad$ Bronze OA & 0.483 & 0.048 & 0.000 & 1.620 \\
$\quad$ Hybrid OA & 0.581 & 0.065 & 0.000 & 1.787 \\
$\quad$ Gold OA & -0.040 & 0.041 & 0.338 & 0.961 \\
No FA vs & & & & \\
$\quad$ Nat FA & 0.247 & 0.027 & 0.000 & 1.280 \\
Int FA & 0.546 & 0.053 & 0.000 & 1.727 \\
$\quad$ EU FA & 0.637 & 0.077 & 0.000 & 1.891 \\
No Coll vs & & & & \\
$\quad$ Nat Coll & 0.220 & 0.031 & 0.000 & 1.246 \\
$\quad$ Int Coll & 0.376 & 0.033 & 0.000 & 1.456 \\
References & 0.012 & 0.001 & 0.000 & 1.012 \\
Usage count & 0.078 & 0.002 & 0.000 & 1.081 \\
Immu vs Econ & 1.282 & 0.029 & 0.000 & 3.604 \\
Constant & -0.999 & 0.035 & 0.000 & 0.368 \\
Nagelkerke R Square & 0.220 & & & \\
\hline OR odds ratio = Exp(B) & & & &
\end{tabular}


This is a postprint version of:

Morillo, F. (2020). Is open access publication useful for all research fields? Presence of funding, collaboration and impact. Scientometrics, 125(1), 689-716.

The final publication is available at Springer via https://doi.org/10.1007/s11192-020-03652-w

Moreover, the probability of finding a cited publication also rises with the interactions between variables (see Appendix). For example, a couple of OA publication types increase the mean predicted probability for Cited thanks to funding (Figure 8). The publications in Bronze $\mathrm{OA}$ are more likely to be cited when they include international funding (OR 1.81, $\mathrm{p}<0.005$ ), while for Green OA papers the probability increases with only national funding (OR 1.47, $p$ $<0.05$ ). Besides, it is interesting to mention that both the Bronze OA publications (OR 1.27, $\mathrm{p}<0.05$ ) and Gold OA items (OR 1.20, $\mathrm{p}<0.05$ ) benefit from collaboration in increasing their citation possibilities (Figure 9), especially international collaboration for Gold OA articles (see Appendix). Finally, it should be also mentioned the great influence that specialisation has, along with some OA types, on the increase of the probability of being cited (Figure 10). This increase is especially significant for Bronze OA when it is published in Immu (OR 1.66, $\mathrm{p}<0.001$ ), but also for Gold OA and Immu (OR 1.61, $\mathrm{p}<0.001$ ).

Figure 8. Mean predicted probability for Cited by OA Types and FA Types

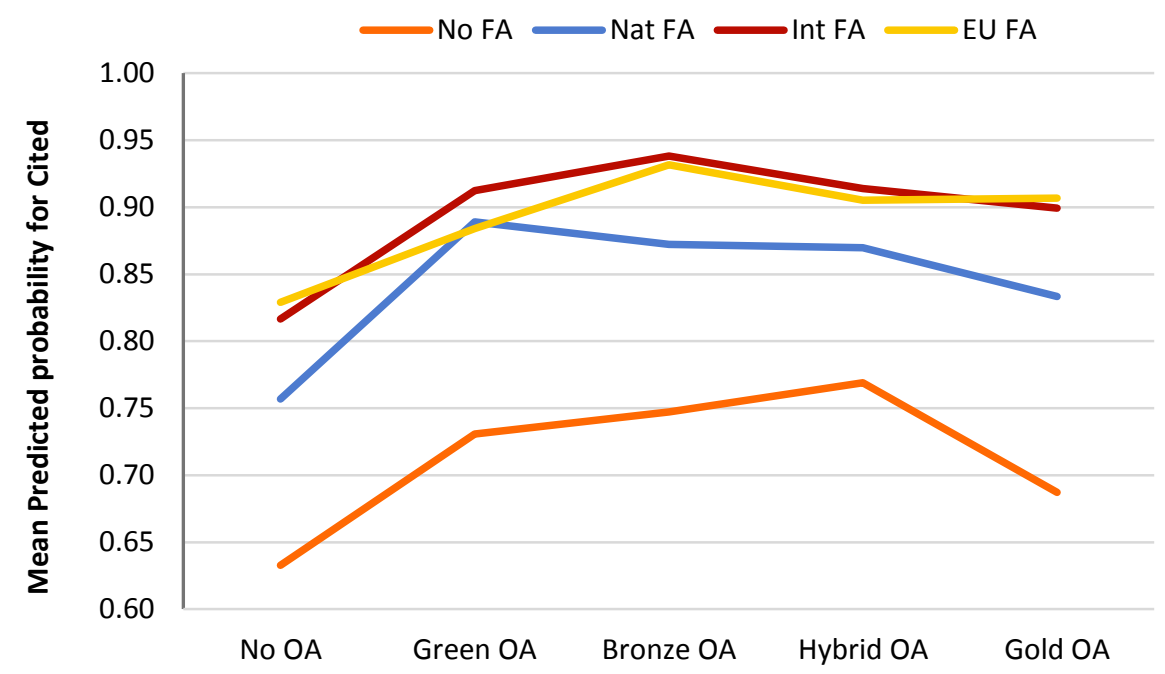

Figure 9. Mean predicted probability for Cited by OA Types and No Coll/Coll

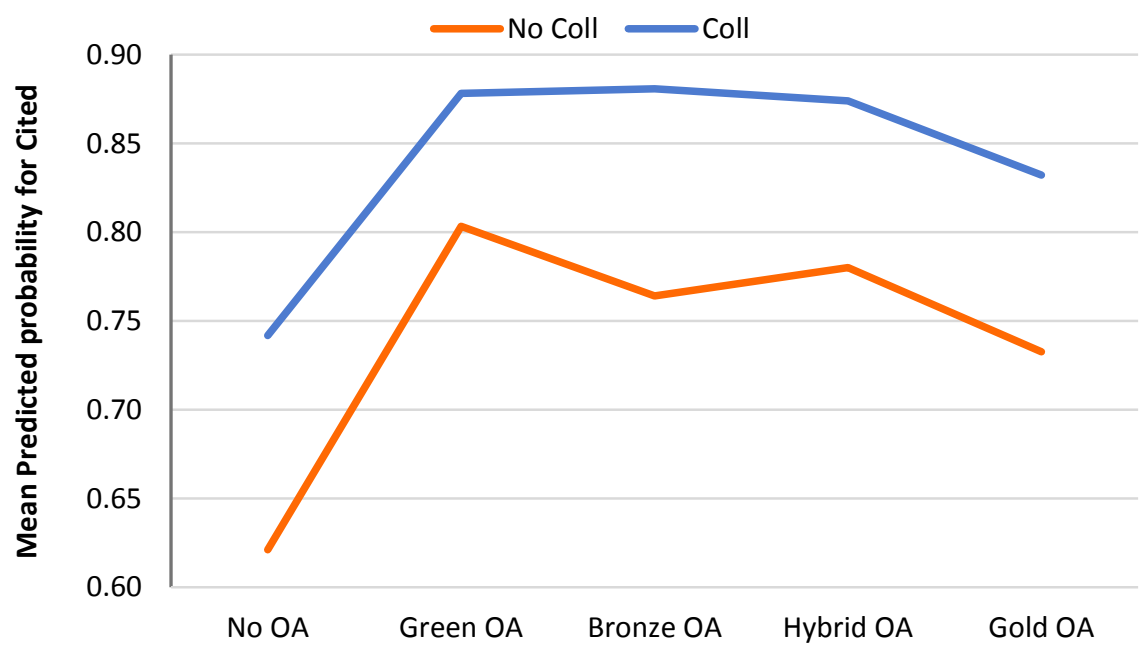


This is a postprint version of:

Morillo, F. (2020). Is open access publication useful for all research fields? Presence of funding, collaboration and impact. Scientometrics, 125(1), 689-716.

The final publication is available at Springer via https://doi.org/10.1007/s11192-020-03652-w

Figure 10. Mean predicted probability for Cited by OA Types and Field

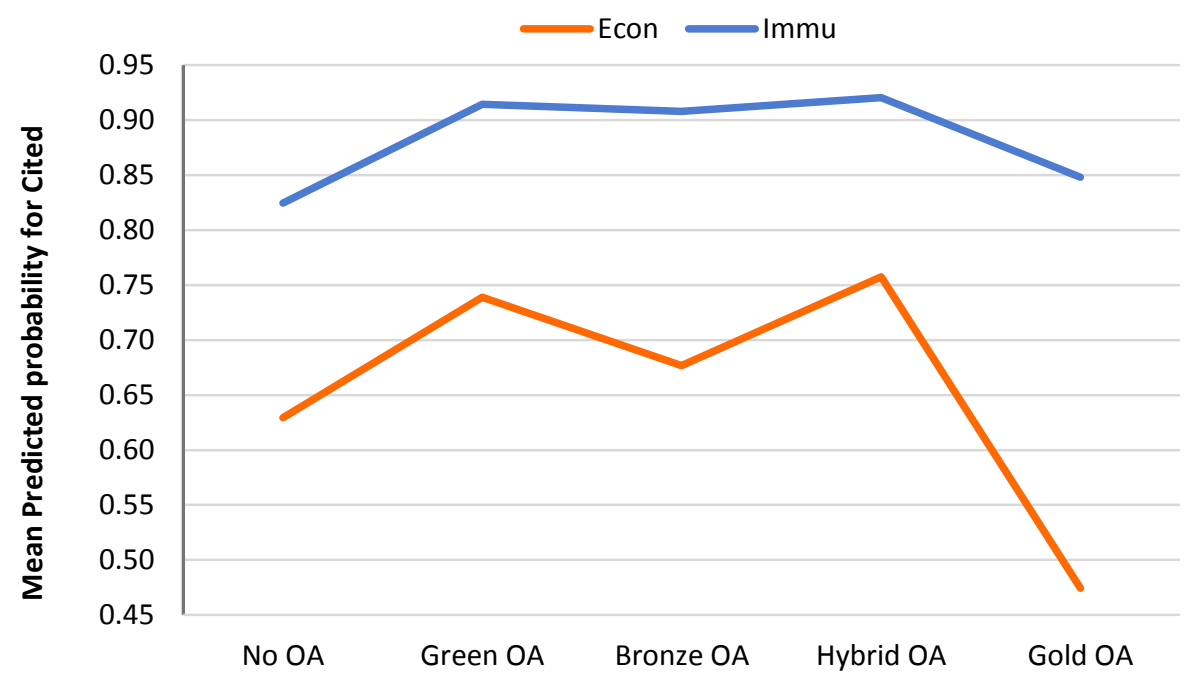

\section{Discussion}

Up to date, the OA publication is still quite scarce, representing around $35 \%$ of the articles written in English in the analysed WoS databases (SCIE and SSCI). Even so, in previous works, a rapid growth of this type of publications has been described, including the years of OA consolidation (e.g. Laakso et al. 2011). The results obtained show that the likelihood of finding an OA publication is related to the presence of FA (mainly from the EU sources, as anticipated in the first hypothesis), collaboration (chiefly international, as supposed in the second hypothesis) and to a greater number of citations (citation advantage), as expected in the third hypothesis. Further to this, the probability for OA rises in particular with some interactions. For instance, $\mathrm{OA}$ articles are more likely when there is collaboration and FA at the same time (especially if both are of international origin), when there are synergies between citations and international FA, or if there is an interaction between international FA and Immu. The last one is clearly influenced by the proportion of this type of funds in Immu. In any case, these connections highlight the importance of funding for OA publishing as predicted in the first hypothesis. This is consistent with the fact that funding agencies include specific requirements regarding the open publication of results sponsored by them and it is the reason why several authors have studied the fulfilment of these requirements (e.g. Larivière and Sugimoto 2018, Pinfield 2010, Wang et al. 2015a).

Additionally, the probability of finding FA in documents increases with Green OA, and with the presence of international collaboration, high citations and research at Immu. Although the Green OA type is partially hidden by the order established in this study, its effect on FA is greater than that of other OA types, probably influenced by Green OA mandates, as stated by Björk et al. 2014. Besides, the occurrence of FA rises with the interaction between OA articles and collaboration, favouring especially Gold OA and Bronze OA items when it is international. This is surprising, considering that the Gold OA publication is the one with the least relation to funding. However, the case of the Gold OA type can be explained by better access to funds through international cooperation, which can be dedicated to support the APC (as 
This is a postprint version of:

Morillo, F. (2020). Is open access publication useful for all research fields? Presence of

funding, collaboration and impact. Scientometrics, 125(1), 689-716.

The final publication is available at Springer via https://doi.org/10.1007/s11192-020-03652-w

Valderrama-Zurián et al. 2019 point out). On the other hand, in the general distribution of documents, it can be seen how the combination between FA and collaboration benefits citation, as it was already detected in previous works, especially for international funds (e.g. Álvarez-Bornstein et al. 2019, Morillo 2019). Moreover, in this study, it has been found that OA publication improves the citation possibilities of this interaction. In fact, Gargouri et al. (2010) find that $O A$ articles obtain higher citation rates regardless of the year, impact factor, number of authors, references, pages, discipline, type of article and country. Piwowar et al. (2018) point out a possible explanation, that greater access to published research may help to obtain a citation advantage, although causality is difficult to establish.

Furthermore, the impact is influenced by OA types. Thus, in the present work, citation likelihood is higher for Hybrid OA publications in line with Eysenbach (2006) and Cintra et al. (2018), since they observe a greater impact on Hybrid OA than on Green OA, although in both of them they find a citation advantage. Besides, the present work includes early citations, which benefit Hybrid OA publications, as they offer faster access. However, in the work by Piwowar et al. (2018) the citation is slightly higher in the Green OA type, followed closely by those of Hybrid OA. Even so, Green OA is somewhat hidden because the other OA types are also put first. For their part, Zhang and Watson (2017) observe that Green OA type is the most common and they do not find significant differences with the citation rates of Gold OA. Nonetheless, in their study, the latter also includes Hybrid OA which, when considered separately, does show higher citation rates. In fact, the last hypothesis of the present study expected that the Gold OA type had less chances of being cited. The results, although not significant, show a lower probability for this OA type, in line with Perianes-Rodríguez and Olmeda-Gómez (2019), who indicate that while the proportion of publications in Gold OA journals increases, the same is not observed for citations. On the other hand, Van Leeuwen et al. (2018) analyse the Gold OA output of three European countries, finding lower impact for the OA results and also a scarce presence of this type of publications ( $10 \%$ at best), although this trend is changing over the years. Similarly, the study by Piwowar et al. (2018) find that Gold OA has a growing presence, although the impact is low and seems to show a decrease, at least in the short term. Notwithstanding, the citation possibilities of certain OA types can experiment an increment with the presence of some interactions. For instance, international collaboration in Gold OA articles shows a higher proportion of citations. This relationship is in line with Breugelmans et al. (2018), because these authors find that OA and international collaboration are important in increasing the impact of publications. Nevertheless, they also find a few exceptions, because cooperation with European countries is not the same as cooperation between sub-Saharan African countries, as the latter not only does not increase the citation rate, but also decreases it.

Apart from that, important differences between disciplines can be observed. Thus, for example, in 2017, the discipline of Econ barely publishes $15 \%$ in OA, while Immu publishes more than $50 \%$ of its articles. These differences between disciplines have also been described previously. For instance, Piwowar et al. (2018) find that biomedical research publishes almost $60 \%$ in OA, especially in the Bronze OA type, although they also observe a high representation for articles published in Gold OA. In the case of the Social Sciences, these authors find a scarce publication in OA (just over 25\%), being the Green OA type the one that obtains the maximum representation. In the present study, and according to the prevalence established in the methodology, the Gold OA type has the greatest presence in Immu, since it accounts for more than $40 \%$ of its publications in OA, although Bronze OA is the second option. In the case of 
This is a postprint version of:

Morillo, F. (2020). Is open access publication useful for all research fields? Presence of

funding, collaboration and impact. Scientometrics, 125(1), 689-716.

The final publication is available at Springer via https://doi.org/10.1007/s11192-020-03652-w

Econ, the latter is the one that reaches the maximum representation of the $O A$, although Hybrid OA and Green OA follow closely. Besides, collaboration is field dependent, because Econ presents less cooperation than Immu, although with higher proportion of international cooperation. Nonetheless, the probability of finding an OA publication increases with international collaboration in Immu, perhaps because of its greater presence of OA and Gold $\mathrm{OA}$ (related to international cooperation as explained above). Another difference between both disciplines is found in the usage count, since Econ shows higher values than Immu. This could be explained by its lower rates of OA publishing, as the usage count seems to be inversely related to open research, possibly because these publications can be accessed from multiple data sources. It should not be forgotten that the usage count is a tool of the WoS database and is limited to the information collected there. Nevertheless, this indicator is directly related to the presence of funding and to the presence of citations, as Chi et al. $\underline{2019}$ already observed.

Regarding impact, Sotudeh and Estakhr (2018) presuppose that citation advantage will not be the same for the different disciplines in the area of Social Sciences and Humanities. The results of the present study corroborate this, since Econ has less citation advantage than Immu, which is related to their different citation habits and to the fact that early citations are included. These differences in citation by discipline are also stated by Norris et al. (2008), as well as the dissimilar percentages of $\mathrm{OA}$ items and the various characteristics of each discipline that probably explain the disparities. Moreover, as revealed in the results, when published in Immu, Bronze OA and Gold OA increase the probability of being cited. This probability may be due to the greater weight of these types in Immu combined with the fact that they offer faster access, thus, there is a knowledge translation advantage, as Eysenbach (2006) states. That is, consumers and policy makers can acquire knowledge in a more accelerated manner. Notwithstanding, although there is some consensus on the OA citation advantage, there is no agreement on the underlying reasons. This is why Sotudeh (2019) decides to study whether citation is related to the topic. She concludes that while there are differences in citation according to the type of research topic, within each of them there is a citation advantage for OA publications, something also confirmed in the present work.

\section{Limitations}

Firstly, the results and conclusions derived from this paper should be framed within the studied research fields and period. Nevertheless, given that two disparate disciplines have been selected, it is possible to venture that the research published in OA will have equally beneficial results for other areas. This advantage of $O A$ is understood in a broad sense, including all types of OA publications.

Secondly, this paper analyses OA articles as collected by the WoS databases, so not everything published in open is taken into account, especially in the cases of Green, Bronze or Hybrid publications. In addition, this study avoids duplication by choosing first the publishers OA types. Therefore, much of the Green OA type is hidden. This also happens in the work by Piwowar et al. (2018), who also indicate that information is lost from some institutional or thematic repositories and point that many authors carry out a retroactive self-archiving, which will increase access to the oldest literature in the future, but not in the present. This loss of Green OA publication is greater if a broader definition is used; covering any location other than that found in institutional repositories, such as departmental or personal web pages (Björk et al. 2014). 
This is a postprint version of:

Morillo, F. (2020). Is open access publication useful for all research fields? Presence of

funding, collaboration and impact. Scientometrics, 125(1), 689-716.

The final publication is available at Springer via https://doi.org/10.1007/s11192-020-03652-w

Thirdly, considering the data source of this study (scientific production collected in WoS), it is not possible to venture anything about the researchers' decisions regarding the publication of their articles. To analyse these decisions, it would be necessary to have supplementary information, which is beyond the scope of this work. What is analysed in this paper is the probability of finding a WoS article published in OA, based on certain characteristics of documents (especially the presence of funding, collaboration and impact).

Fourthly, a relatively recent year has been selected to obtain a greater proportion of OA articles, since in previous works it has been found that this increases over time (e.g. Piwowar et al. 2018). For this reason, as citations take time to occur, it must be considered that part of the ones studied here are very recent and that this negatively affects the research carried out in fields that use older literature. Nonetheless, it is interesting to examine to what extent the OA publication facilitates faster access to research results and their subsequent recognition through citations.

Finally, regarding the study of FA, limitations of the acknowledgements collected in publications must be taken into account, because they do not always include all the monetary support received and are only a partial result of the research carried out (as pointed out by Morillo and Álvarez-Bornstein 2018).

\section{Conclusion}

The findings obtained in the present study highlight the usefulness of OA publishing in accelerating access to quality research that serves to produce new knowledge. They also demonstrate that $\mathrm{OA}$ is related to greater funding, collaboration and impact of research. Although it is difficult to determine the cause-effect, greater funding and collaboration can encourage OA publishing and both can favour the citation advantage. However, other factors are expected to come into play once the OA becomes more represented. In any case, it is important to take into account the differences between research fields, both in OA publishing habits and in the support received to increase this access. In fact, in social sciences and humanities, the OA journals offer remains scarce and tends to have less impact. Funding bodies should be aware of these particularities when articulating their measures to stimulate the $\mathrm{OA}$ to new knowledge and encourage the creation of resources for the dissemination of information without compromising the quality of research.

\section{Acknowledgements}

This work is partially supported by the Spanish Ministry of Economy and Competitiveness (Grant CSO2014-57826-P). 
This is a postprint version of:

Morillo, F. (2020). Is open access publication useful for all research fields? Presence of funding, collaboration and impact. Scientometrics, 125(1), 689-716.

The final publication is available at Springer via https://doi.org/10.1007/s11192-020-03652-w

\section{Appendix}

See Table 6, Table 7, Table 8, Table 9 and Table 10.

Table 6. Logistic regression for the OA presence/absence with some interactions between variables

\begin{tabular}{|c|c|c|c|c|c|c|c|c|c|c|c|c|}
\hline & \multicolumn{4}{|c|}{$\begin{array}{l}\text { Model 2: FA Types * } \\
\text { Coll Types }\end{array}$} & \multicolumn{4}{|c|}{$\begin{array}{c}\text { Model 3: FA Types * } \\
\text { Cited }\end{array}$} & \multicolumn{4}{|c|}{$\begin{array}{l}\text { Model 4: FA Types * } \\
\text { Field }\end{array}$} \\
\hline & B & S.E. & Sig. & OR & B & S.E. & Sig. & OR & B & S.E. & Sig. & OR \\
\hline \multicolumn{13}{|l|}{ No FA vs. } \\
\hline Nat FA & 0.542 & 0.054 & 0.000 & 1.720 & 0.503 & 0.054 & 0.000 & 1.654 & 0.301 & 0.044 & 0.000 & 1.352 \\
\hline Int FA & 0.398 & 0.136 & 0.003 & 1.489 & 0.528 & 0.107 & 0.000 & 1.696 & 0.408 & 0.079 & 0.000 & 1.504 \\
\hline EU FA & 0.825 & 0.156 & 0.000 & 2.281 & 1.025 & 0.151 & 0.000 & 2.786 & 0.729 & 0.092 & 0.000 & 2.074 \\
\hline No Coll vs. & & & 0.000 & & & & & & & & & \\
\hline Nat Coll & -0.081 & 0.052 & 0.122 & 0.922 & 0.021 & 0.031 & 0.493 & 1.022 & 0.022 & 0.031 & 0.477 & 1.022 \\
\hline Int Coll & 0.140 & 0.059 & 0.018 & 1.151 & 0.327 & 0.033 & 0.000 & 1.387 & 0.303 & 0.033 & 0.000 & 1.354 \\
\hline Cited & & & & & 0.140 & 0.049 & 0.004 & 1.150 & & & & \\
\hline No cited vs. & & & & & & & & & & & 0.000 & \\
\hline Low cited & 0.057 & 0.034 & 0.090 & 1.059 & & & & & 0.056 & 0.034 & 0.097 & 1.058 \\
\hline Medium cited & 0.318 & 0.035 & 0.000 & 1.374 & & & & & 0.312 & 0.035 & 0.000 & 1.366 \\
\hline Highly cited & 0.611 & 0.036 & 0.000 & 1.842 & & & & & 0.612 & 0.036 & 0.000 & 1.845 \\
\hline References & 0.003 & 0.000 & 0.000 & 1.003 & 0.003 & 0.000 & 0.000 & 1.003 & 0.002 & 0.000 & 0.000 & 1.002 \\
\hline Usage count & -0.026 & 0.002 & 0.000 & 0.975 & -0.020 & 0.001 & 0.000 & 0.980 & -0.026 & 0.002 & 0.000 & 0.974 \\
\hline Immu vs. Econ & 1.512 & 0.027 & 0.000 & 4.535 & 1.489 & 0.027 & 0.000 & 4.432 & 1.117 & 0.044 & 0.000 & 3.055 \\
\hline \multicolumn{13}{|l|}{ FA Types * Coll Types } \\
\hline Nat FA by Nat Coll & 0.125 & 0.066 & 0.057 & 1.133 & & & & & & & & \\
\hline Nat FA by Int Coll & 0.189 & 0.074 & 0.011 & 1.208 & & & & & & & & \\
\hline Int FA by Nat Coll & 0.407 & 0.156 & 0.009 & 1.502 & & & & & & & & \\
\hline Int FA by Int Coll & 0.534 & 0.148 & 0.000 & 1.706 & & & & & & & & \\
\hline EU FA by Nat Coll & 0.225 & 0.190 & 0.235 & 1.253 & & & & & & & & \\
\hline EU FA by Int Coll & 0.342 & 0.175 & 0.050 & 1.408 & & & & & & & & \\
\hline \multicolumn{13}{|l|}{ FA Types * Cited } \\
\hline Nat FA by Cited & & & & & 0.212 & 0.061 & 0.001 & 1.236 & & & & \\
\hline Int FA by Cited & & & & & 0.400 & 0.115 & 0.000 & 1.492 & & & & \\
\hline EU FA by Cited & & & & & 0.118 & 0.162 & 0.468 & 1.125 & & & & \\
\hline \multicolumn{13}{|l|}{ FA Types * Field } \\
\hline Nat FA by Immu & & & & & & & & & 0.579 & 0.056 & 0.000 & 1.784 \\
\hline Int FA by Immu & & & & & & & & & 0.659 & 0.092 & 0.000 & 1.933 \\
\hline EU FA by Immu & & & & & & & & & 0.590 & 0.116 & 0.000 & 1.805 \\
\hline Constant & -2.304 & 0.047 & 0.000 & 0.100 & -2.341 & 0.047 & 0.000 & 0.096 & -2.180 & 0.042 & 0.000 & 0.113 \\
\hline Nagelkerke R Square & 0.243 & & & & 0.235 & & & & 0.246 & & & \\
\hline
\end{tabular}

OR odds ratio $=\operatorname{Exp}(B)$

Table 7. Logistic regression for the OA presence/absence with interactions between Coll Types and Field

\begin{tabular}{lcccc}
\hline & \multicolumn{4}{c}{ Model 4: Coll Types * Field } \\
\cline { 2 - 5 } & B & S.E. & Sig. & OR \\
\hline No FA vs. & & & & \\
Nat FA & 0.652 & 0.027 & 0.000 & 1.920 \\
Int FA & 0.808 & 0.043 & 0.000 & 2.243 \\
EU FA & 1.058 & 0.057 & 0.000 & 2.880 \\
No Coll vs. & & & 0.007 & \\
\hline
\end{tabular}


This is a postprint version of:

Morillo, F. (2020). Is open access publication useful for all research fields? Presence of funding, collaboration and impact. Scientometrics, 125(1), 689-716.

The final publication is available at Springer via https://doi.org/10.1007/s11192-020-03652-w

\begin{tabular}{lclll}
\hline & \multicolumn{4}{c}{ Model 4: Coll Types * Field } \\
\cline { 2 - 5 } & B & S.E. & Sig. & OR \\
\hline Nat Coll & 0.012 & 0.053 & 0.819 & 1.012 \\
Int Coll & 0.139 & 0.050 & 0.006 & 1.149 \\
No cited vs. & & & & \\
$\quad$ Low cited & 0.058 & 0.034 & 0.084 & 1.060 \\
$\quad$ Medium cited & 0.318 & 0.035 & 0.000 & 1.374 \\
$\quad$ Highly cited & 0.611 & 0.036 & 0.000 & 1.842 \\
References & 0.003 & 0.000 & 0.000 & 1.003 \\
Usage count & -0.026 & 0.002 & 0.000 & 0.975 \\
Immu vs. Econ & 1.396 & 0.052 & 0.000 & 4.041 \\
Coll Types * Field & & & & \\
$\quad$ Nat Coll by Immu & 0.036 & 0.065 & 0.584 & 1.036 \\
$\quad$ Int Coll by Immu & 0.267 & 0.065 & 0.000 & 1.306 \\
Constant & -2.322 & 0.045 & 0.000 & 0.098 \\
Nagelkerke R Square & 0.243 & & & \\
OR odds ratio = Exp(B) & & & &
\end{tabular}

Table 8. Logistic regression for the FA presence/absence with some interactions between variables

\begin{tabular}{|c|c|c|c|c|c|c|c|c|c|c|c|c|}
\hline & \multicolumn{4}{|c|}{$\begin{array}{l}\text { Model 2: OA Types * } \\
\text { Coll Types }\end{array}$} & \multicolumn{4}{|c|}{$\begin{array}{c}\text { Model 3: OA Types * } \\
\text { Cited }\end{array}$} & \multicolumn{4}{|c|}{$\begin{array}{l}\text { Model 4: OA Types * } \\
\text { Field }\end{array}$} \\
\hline & B & S.E. & Sig. & OR & B & S.E. & Sig. & OR & B & S.E. & Sig. & OR \\
\hline \multicolumn{13}{|l|}{ No OA vs. } \\
\hline Green OA & 0.721 & 0.131 & 0.000 & 2.057 & 0.654 & 0.132 & 0.000 & 1.923 & 0.496 & 0.088 & 0.000 & 1.642 \\
\hline Bronze OA & 0.581 & 0.087 & 0.000 & 1.787 & 0.617 & 0.092 & 0.000 & 1.853 & 0.291 & 0.070 & 0.000 & 1.338 \\
\hline Hybrid OA & 0.675 & 0.116 & 0.000 & 1.965 & 0.773 & 0.124 & 0.000 & 2.166 & 0.573 & 0.074 & 0.000 & 1.773 \\
\hline Gold OA & 0.452 & 0.074 & 0.000 & 1.571 & 0.287 & 0.072 & 0.000 & 1.332 & -0.043 & 0.093 & 0.644 & 0.958 \\
\hline No Coll vs. & & & 0.000 & & & & & & & & 0.000 & \\
\hline Nat Coll & 0.432 & 0.031 & 0.000 & 1.541 & 0.451 & 0.027 & 0.000 & 1.570 & 0.451 & 0.027 & 0.000 & 1.570 \\
\hline Int Coll & 0.751 & 0.033 & 0.000 & 2.120 & 0.821 & 0.029 & 0.000 & 2.273 & 0.803 & 0.029 & 0.000 & 2.232 \\
\hline Cited & & & & & 0.300 & 0.028 & 0.000 & 1.350 & & & & \\
\hline \multicolumn{13}{|l|}{ No cited vs. } \\
\hline Low cited & 0.223 & 0.030 & 0.000 & 1.250 & & & & & 0.220 & 0.030 & 0.000 & 1.246 \\
\hline Medium cited & 0.425 & 0.033 & 0.000 & 1.529 & & & & & 0.417 & 0.033 & 0.000 & 1.517 \\
\hline Highly cited & 0.470 & 0.033 & 0.000 & 1.599 & & & & & 0.456 & 0.033 & 0.000 & 1.577 \\
\hline Usage count & 0.013 & 0.001 & 0.000 & 1.013 & 0.015 & 0.001 & 0.000 & 1.015 & 0.013 & 0.001 & 0.000 & 1.013 \\
\hline Immu vs. Econ & 1.152 & 0.025 & 0.000 & 3.165 & 1.159 & 0.025 & 0.000 & 3.187 & 1.007 & 0.027 & 0.000 & 2.736 \\
\hline \multicolumn{13}{|l|}{ OA Types * Coll Types } \\
\hline Green OA by Nat Coll & 0.549 & 0.167 & 0.001 & 1.731 & & & & & & & & \\
\hline Green OA by Int Coll & 0.124 & 0.162 & 0.442 & 1.133 & & & & & & & & \\
\hline Bronze OA by Nat Coll & 0.185 & 0.106 & 0.080 & 1.203 & & & & & & & & \\
\hline Bronze OA by Int Coll & 0.350 & 0.117 & 0.003 & 1.419 & & & & & & & & \\
\hline Hybrid OA by Nat Coll & 0.136 & 0.147 & 0.354 & 1.146 & & & & & & & & \\
\hline Hybrid OA by Int Coll & 0.139 & 0.146 & 0.341 & 1.149 & & & & & & & & \\
\hline Gold OA by Nat Coll & -0.083 & 0.088 & 0.344 & 0.920 & & & & & & & & \\
\hline Gold OA by Int Coll & 0.392 & 0.109 & 0.000 & 1.479 & & & & & & & & \\
\hline \multicolumn{13}{|l|}{ OA Types * Cited } \\
\hline Green OA by Cited & & & & & 0.441 & 0.149 & 0.003 & 1.554 & & & & \\
\hline Bronze OA by Cited & & & & & 0.224 & 0.103 & 0.029 & 1.251 & & & & \\
\hline Hybrid OA by Cited & & & & & 0.051 & 0.139 & 0.711 & 1.053 & & & & \\
\hline Gold OA by Cited & & & & & 0.279 & 0.083 & 0.001 & 1.321 & & & & \\
\hline \multicolumn{13}{|l|}{ OA Types * Field } \\
\hline Green OA by Immu & & & & & & & & & 0.965 & 0.129 & 0.000 & 2.624 \\
\hline Bronze OA by Immu & & & & & & & & & 0.792 & 0.090 & 0.000 & 2.209 \\
\hline
\end{tabular}


This is a postprint version of:

Morillo, F. (2020). Is open access publication useful for all research fields? Presence of funding, collaboration and impact. Scientometrics, 125(1), 689-716.

The final publication is available at Springer via https://doi.org/10.1007/s11192-020-03652-w

\begin{tabular}{|c|c|c|c|c|c|c|c|c|c|c|c|c|}
\hline & \multicolumn{4}{|c|}{$\begin{array}{l}\text { Model 2: OA Types* } \\
\text { Coll Types }\end{array}$} & \multicolumn{4}{|c|}{$\begin{array}{c}\text { Model 3: OA Types * } \\
\text { Cited }\end{array}$} & \multicolumn{4}{|c|}{$\begin{array}{l}\text { Model 4: OA Types * } \\
\text { Field }\end{array}$} \\
\hline & B & S.E. & Sig. & OR & B & S.E. & Sig. & OR & B & S.E. & Sig. & OR \\
\hline Hybrid OA by Immu & & & & & & & & & 0.494 & 0.114 & 0.000 & 1.639 \\
\hline Gold OA by Immu & & & & & & & & & 0.707 & 0.103 & 0.000 & 2.029 \\
\hline Constant & -0.959 & 0.030 & 0.000 & 0.383 & -0.970 & 0.030 & 0.000 & 0.379 & -0.923 & 0.029 & 0.000 & 0.397 \\
\hline Nagelkerke R Square & 0.208 & & & & 0.205 & & & & 0.211 & & & \\
\hline
\end{tabular}

Table 9. Logistic regression for the presence/absence of citations with some interactions between variables

\begin{tabular}{|c|c|c|c|c|c|c|c|c|c|c|c|c|}
\hline & \multicolumn{4}{|c|}{$\begin{array}{c}\text { Model 2: OA Types * } \\
\text { FA Types }\end{array}$} & \multicolumn{4}{|c|}{$\begin{array}{c}\text { Model 4: OA Types * } \\
\text { Coll }\end{array}$} & \multicolumn{4}{|c|}{$\begin{array}{l}\text { Model 3: OA Types * } \\
\text { Field }\end{array}$} \\
\hline & B & S.E. & Sig. & OR & B & S.E. & Sig. & OR & B & S.E. & Sig. & OR \\
\hline \multicolumn{13}{|l|}{ No OA vs. } \\
\hline Green OA & 0.257 & 0.129 & 0.046 & 1.293 & 0.373 & 0.156 & 0.017 & 1.452 & 0.309 & 0.100 & 0.002 & 1.362 \\
\hline Bronze OA & 0.323 & 0.087 & 0.000 & 1.381 & 0.319 & 0.099 & 0.001 & 1.376 & 0.187 & 0.077 & 0.015 & 1.206 \\
\hline Hybrid OA & 0.538 & 0.117 & 0.000 & 1.712 & 0.496 & 0.137 & 0.000 & 1.642 & 0.480 & 0.084 & 0.000 & 1.616 \\
\hline Gold OA & -0.111 & 0.070 & 0.115 & 0.895 & -0.170 & 0.083 & 0.040 & 0.844 & -0.390 & 0.094 & 0.000 & 0.677 \\
\hline FA & & & & & 0.311 & 0.026 & 0.000 & 1.365 & & & & \\
\hline \multicolumn{13}{|l|}{ No FA vs. } \\
\hline Nat FA & 0.217 & 0.030 & 0.000 & 1.243 & & & & & 0.239 & 0.027 & 0.000 & 1.270 \\
\hline Int FA & 0.451 & 0.064 & 0.000 & 1.570 & & & & & 0.533 & 0.054 & 0.000 & 1.705 \\
\hline EU FA & 0.634 & 0.096 & 0.000 & 1.886 & & & & & 0.630 & 0.077 & 0.000 & 1.878 \\
\hline Coll & & & & & 0.263 & 0.031 & 0.000 & 1.301 & & & & \\
\hline \multicolumn{13}{|l|}{ No Coll vs. } \\
\hline Nat Coll & 0.222 & 0.031 & 0.000 & 1.248 & & & & & 0.221 & 0.031 & 0.000 & 1.247 \\
\hline Int Coll & 0.375 & 0.033 & 0.000 & 1.455 & & & & & 0.370 & 0.033 & 0.000 & 1.448 \\
\hline References & 0.012 & 0.001 & 0.000 & 1.012 & 0.012 & 0.001 & 0.000 & 1.012 & 0.012 & 0.001 & 0.000 & 1.012 \\
\hline Usage count & 0.078 & 0.002 & 0.000 & 1.082 & 0.079 & 0.002 & 0.000 & 1.082 & 0.078 & 0.002 & 0.000 & 1.081 \\
\hline Immu vs. Econ & 1.275 & 0.029 & 0.000 & 3.579 & 1.245 & 0.028 & 0.000 & 3.474 & 1.184 & 0.032 & 0.000 & 3.267 \\
\hline OA Types* FA Types & & & 0.045 & & & & & & & & & \\
\hline Green OA by Nat FA & 0.383 & 0.157 & 0.015 & 1.466 & & & & & & & & \\
\hline Green OA by Int FA & 0.275 & 0.237 & 0.246 & 1.316 & & & & & & & & \\
\hline Green OA by EU FA & -0.227 & 0.318 & 0.477 & 0.797 & & & & & & & & \\
\hline Bronze OA by Nat FA & 0.175 & 0.107 & 0.101 & 1.191 & & & & & & & & \\
\hline Bronze OA by Int FA & 0.594 & 0.190 & 0.002 & 1.812 & & & & & & & & \\
\hline Bronze OA by EU FA & 0.340 & 0.287 & 0.236 & 1.404 & & & & & & & & \\
\hline Hybrid OA by Nat FA & 0.087 & 0.147 & 0.554 & 1.091 & & & & & & & & \\
\hline Hybrid OA by Int FA & 0.119 & 0.239 & 0.619 & 1.126 & & & & & & & & \\
\hline Hybrid OA by EU FA & -0.091 & 0.277 & 0.743 & 0.913 & & & & & & & & \\
\hline Gold OA by Nat FA & 0.096 & 0.086 & 0.267 & 1.100 & & & & & & & & \\
\hline Gold OA by Int FA & 0.263 & 0.161 & 0.102 & 1.301 & & & & & & & & \\
\hline Gold OA by EU FA & 0.156 & 0.235 & 0.507 & 1.169 & & & & & & & & \\
\hline \multicolumn{13}{|l|}{ OA Types * Coll } \\
\hline Green OA by Coll & & & & & 0.184 & 0.172 & 0.285 & 1.202 & & & & \\
\hline Bronze OA by Coll & & & & & 0.239 & 0.112 & 0.032 & 1.271 & & & & \\
\hline Hybrid OA by Coll & & & & & 0.163 & 0.155 & 0.293 & 1.177 & & & & \\
\hline Gold OA by Coll & & & & & 0.182 & 0.092 & 0.049 & 1.199 & & & & \\
\hline \multicolumn{13}{|l|}{ OA Types * Field } \\
\hline Green OA by Immu & & & & & & & & & 0.366 & 0.135 & 0.007 & 1.441 \\
\hline Bronze OA by Immu & & & & & & & & & 0.505 & 0.099 & 0.000 & 1.657 \\
\hline Hybrid OA by Immu & & & & & & & & & 0.268 & 0.132 & 0.042 & 1.308 \\
\hline
\end{tabular}


This is a postprint version of:

Morillo, F. (2020). Is open access publication useful for all research fields? Presence of funding, collaboration and impact. Scientometrics, 125(1), 689-716.

The final publication is available at Springer via https://doi.org/10.1007/s11192-020-03652-w

\begin{tabular}{|c|c|c|c|c|c|c|c|c|c|c|c|c|}
\hline & \multicolumn{4}{|c|}{$\begin{array}{c}\text { Model 2: OA Types * } \\
\text { FA Types }\end{array}$} & \multicolumn{4}{|c|}{$\begin{array}{c}\text { Model 4: OA Types* } \\
\text { Coll }\end{array}$} & \multicolumn{4}{|c|}{$\begin{array}{l}\text { Model 3: OA Types * } \\
\text { Field }\end{array}$} \\
\hline & B & S.E. & Sig. & OR & B & S.E. & Sig. & OR & B & S.E. & Sig. & OR \\
\hline Gold OA by Immu & & & & & & & & & 0.478 & 0.105 & 0.000 & 1.613 \\
\hline Constant & -0.979 & 0.036 & 0.000 & 0.376 & -1.301 & 0.048 & 0.000 & 0.272 & -0.960 & 0.035 & 0.000 & 0.383 \\
\hline Nagelkerke R Square & 0.221 & & & & 0.217 & & & & 0.222 & & & \\
\hline
\end{tabular}

OR odds ratio $=\operatorname{Exp}(B)$

Table 10. Logistic regression for the presence/absence of citations with interactions between OA Types and Coll Types

\begin{tabular}{lcccc}
\hline & \multicolumn{4}{c}{ Model 4a: OA Types * Coll Types } \\
\cline { 2 - 5 } & B & S.E. & Sig. & OR \\
\hline No OA vs. & & & & \\
Green OA & 0.373 & 0.156 & 0.017 & 1.453 \\
Bronze OA & 0.318 & 0.099 & 0.001 & 1.375 \\
Hybrid OA & 0.492 & 0.137 & 0.000 & 1.636 \\
Gold OA & -0.177 & 0.083 & 0.032 & 0.838 \\
No FA vs. & & & & \\
Nat FA & 0.245 & 0.027 & 0.000 & 1.278 \\
Int FA & 0.539 & 0.054 & 0.000 & 1.714 \\
EU FA & 0.634 & 0.077 & 0.000 & 1.885 \\
No Coll vs. & & & & \\
$\quad$ Nat Coll & 0.183 & 0.034 & 0.000 & 1.201 \\
Int Coll & 0.340 & 0.037 & 0.000 & 1.404 \\
References & 0.012 & 0.001 & 0.000 & 1.012 \\
Usage count & 0.078 & 0.002 & 0.000 & 1.082 \\
Immu vs. Econ & 1.279 & 0.029 & 0.000 & 3.591 \\
OA Types $*$ Coll Types & & & & \\
Green OA by Nat Coll & 0.328 & 0.190 & 0.085 & 1.388 \\
Green OA by Int Coll & -0.022 & 0.186 & 0.906 & 0.978 \\
Bronze OA by Nat Coll & 0.213 & 0.120 & 0.077 & 1.237 \\
Bronze OA by Int Coll & 0.220 & 0.130 & 0.091 & 1.246 \\
Hybrid OA by Nat Coll & 0.118 & 0.172 & 0.493 & 1.125 \\
Hybrid OA by Int Coll & 0.118 & 0.171 & 0.490 & 1.125 \\
Gold OA by Nat Coll & 0.142 & 0.097 & 0.145 & 1.152 \\
Gold OA by Int Coll & 0.272 & 0.114 & 0.017 & 1.313 \\
Constant & -0.972 & 0.036 & 0.000 & 0.378 \\
Nagelkerke R Square & 0.221 & & & \\
\hline OR odds rati = Exp(B) & & & & \\
\hline
\end{tabular}

OR odds ratio $=\operatorname{Exp}(B)$

\section{References}

Álvarez-Bornstein, B., Díaz-Faes, A. A., \& Bordons, M. (2019). What characterises funded biomedical research? Evidence from a basic and a clinical domain. Scientometrics, 119(2), 805-825. doi:10.1007/s11192-019-03066-3

Antelman, K. (2004). Do open-access articles have a greater research impact?. College \& research libraries, 65(5), 372-382. doi:10.5860/crl.65.5.372

Björk, B. C. (2017). Growth of hybrid open access, 2009-2016. PeerJ, 5, e3878. doi:10.7717/peeri.3878 
This is a postprint version of:

Morillo, F. (2020). Is open access publication useful for all research fields? Presence of

funding, collaboration and impact. Scientometrics, 125(1), 689-716.

The final publication is available at Springer via https://doi.org/10.1007/s11192-020-03652-w

Björk, B. C., \& Solomon, D. (2015). Article processing charges in OA journals: relationship between price and quality. Scientometrics, 103(2), 373-385. doi:10.1007/s11192-015-1556-z

Björk, B. C., Laakso, M., Welling, P., \& Paetau, P. (2014). Anatomy of green open access. Journal of the Association for Information Science and Technology, 65(2), 237-250. doi:10.1002/asi.22963

Breugelmans, J. G., Roberge, G., Tippett, C., Durning, M., Struck, D. B., \& Makanga, M. M. (2018). Scientific impact increases when researchers publish in open access and international collaboration: A bibliometric analysis on poverty-related disease papers. PLOS ONE, 13(9), e0203156. doi:10.1371/journal.pone.0203156

Chi, P. S., Gorraiz, J., \& Glänzel, W. (2019). Comparing capture, usage and citation indicators: an altmetric analysis of journal papers in chemistry disciplines. Scientometrics, 120(3), 1461-1473. doi:10.1007/s11192-019-03168-y

Cintra, P. R., Furnival, A. C., \& Milanez, D. H. (2018). The impact of open access citation and social media on leading top Information Science journals. INVESTIGACIÓN BIBLIOTECOLÓGICA, 32(77), 117-132. doi:10.22201/iibi.24488321xe.2018.77.57874

Else, H. (2018). Radical open-access plan could spell end to journal subscriptions. Nature, 561(7721), 17-18. doi:10.1038/d41586-018-06178-7

Eysenbach, G. (2006). Citation advantage of open access articles. PLoS Biology, 4(5), e157. doi:10.1371/journal.pbio.0040157

Gargouri, Y., Hajjem, C., Larivière, V., Gingras, Y., Carr, L., Brody, T., \& Harnad, S. (2010). Self-selected or mandated, open access increases citation impact for higher quality research. PLOS ONE, 5(10), e13636. doi:10.1371/journal.pone.0013636

Khoo, S. (2019). Article Processing Charge Hyperinflation and Price Insensitivity: An Open Access Sequel to the Serials Crisis. LIBER Quarterly, 29(1), 1-18. doi:10.18352/lq.10280

Laakso, M., Welling, P., Bukvova, H., Nyman, L., Björk, B. C., \& Hedlund, T. (2011). The development of open access journal publishing from 1993 to 2009. PLOS ONE, 6(6), e20961. doi:10.1371/journal.pone.0020961

Larivière, V., \& Sugimoto, C. R. (2018). Do authors comply when funders enforce open access to research?. Nature, 562(7728), 483-486. doi:10.1038/d41586-018-07101-w

Martín-Martín, A., Costas, R., van Leeuwen, T., \& López-Cózar, E. D. (2018). Evidence of open access of scientific publications in Google Scholar: A large-scale analysis. Journal of Informetrics, 12(3), 819-841. doi:10.1016/i.joi.2018.06.012

Morillo, F. (2019). Collaboration and impact of research in different disciplines with international funding (from the EU and other foreign sources). Scientometrics, 120(2), 807-823. doi:10.1007/s11192-019-03150-8

Morillo, F., \& Álvarez-Bornstein, B. (2018). How to automatically identify major research sponsors selecting keywords from the WoS Funding Agency field. Scientometrics, 117(3), 1755-1770. doi:10.1007/s11192-018-2947-8.

Norris, M., Oppenheim, C., \& Rowland, F. (2008). The citation advantage of open-access articles. Journal of the American Society for Information Science and Technology, 59(12), 1963-1972. doi:10.1002/asi.20898

Perianes-Rodríguez, A., \& Olmeda-Gómez, C. (2019). Effects of journal choice on the visibility of scientific publications: a comparison between subscription-based and full Open Access models. Scientometrics, 121(3), 1737-1752. doi:10.1007/s11192-019-03265-y

Pinfield, S. (2010). Paying for open access? Institutional funding streams and OA publication charges. Learned Publishing, 23(1), 39-52. doi:10.1087/20100108 
This is a postprint version of:

Morillo, F. (2020). Is open access publication useful for all research fields? Presence of funding, collaboration and impact. Scientometrics, 125(1), 689-716.

The final publication is available at Springer via https://doi.org/10.1007/s11192-020-03652-w

Piwowar, H., Priem, J., Larivière, V., Alperin, J. P., Matthias, L., Norlander, B., Farley, A., West, J. \& Haustein, S. (2018). The State of OA: A large-scale analysis of the prevalence and impact of Open Access articles. PeerJ, 6, e4375. doi:10.7717/peerj.4375

Sotudeh, H. (2019). Does open access citation advantage depend on paper topics?. Journal of Information Science. First published online July 22, 2019. doi:10.1177/2F0165551519865489

Sotudeh, H., \& Estakhr, Z. (2018). Sustainability of open access citation advantage: the case of Elsevier's author-pays hybrid open access journals. Scientometrics, 115(1), 563-576. doi:10.1007/s11192-018-2663-4

Valderrama-Zurián, J. C., Aguilar-Moya, R., \& Gorraiz, J. (2019). On the bibliometric nature of a foreseeable relationship: open access and education. Scientometrics, 120(3), 1031-1057. doi:10.1007/s11192-019-03175-z

Van Leeuwen, T. N., Tatum, C., \& Wouters, P. F. (2018). Exploring possibilities to use bibliometric data to monitor gold open access publishing at the national level. Journal of the Association for Information Science and Technology, 69(9), 1161-1173. doi:10.1002/asi.24029

Van Vlokhoven, H. (2019). The effect of open access on research quality. Journal of Informetrics, 13(2), 751-756. doi:10.1016/i.joi.2019.04.001

Wang, L. L., Liu, X. Z., \& Fang, H. (2015a). Investigation of the degree to which articles supported by research grants are published in open access health and life sciences journals. Scientometrics, 104(2), 511-528. doi:10.1007/s11192-015-1624-4

Wang, X., Liu, C., Mao, W., \& Fang, Z. (2015b). The open access advantage considering citation, article usage and social media attention. Scientometrics, 103(2), 555-564. doi:10.1007/s11192-015-1547-0

Zhang, L., \& Watson, E. M. (2017). Measuring the impact of gold and green open access. The Journal of Academic Librarianship, 43(4), 337-345. doi:10.1016/i.acalib.2017.06.004 